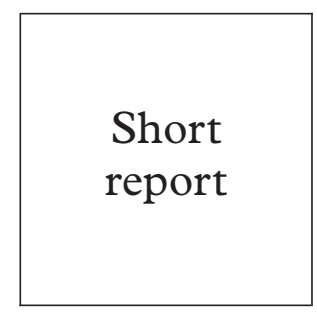

\title{
Knowledge, attitudes, and practices regarding sexually transmitted infections among general practitioners and medical specialists in Karachi, Pakistan
}

\author{
H E Khandwalla, S Luby, S Rahman
}

Objectives: To determine the knowledge, attitudes, and practices regarding diagnosis and treatment of sexually transmitted infections (STIs) among specialists - that is, dermatologists, gynaecologists and urologists, and general practitioners (GPs) in Karachi, Pakistan.

Methods: Interviewers administered structured questionnaires to doctors conducting outpatient clinics at tertiary hospitals and/or private clinics in Karachi. All private clinics within a $10 \mathrm{~km}$ radius of the Aga Khan University, and all tertiary hospitals having more than 100 inpatient beds were included in the study.

Results: 100 doctors (54 specialists and 46 GPs) responded. 80 doctors reported seeing at least one STI patient/month. The most commonly diagnosed STI the doctors reported was urethritis/ cervicitis syndrome. $50 \%$ of the doctors knew the recommended antibiotics for gonorrhoea though only $46 \%$ of these knew the correct dosage. Specialists were three times more likely to recognise the clinical presentation of herpes and twice as likely to treat chlamydia, syphilis, and herpes with appropriate antimicrobials than GPs. $85 \%$ of the doctors advised their STI patients regarding condom usage; $36 \%$ thought that STI patients had loose sexual morals; $43 \%$ believed STI patients were drug addicts. Over $90 \%$ of the physicians were willing to attend educational sessions and follow a national STI treatment protocol.

Conclusion: Doctors in Karachi, especially GPs, are deficient in appropriately managing and counselling STI patients. Among the specialists, urologists and dermatologists were more likely to manage STIs correctly than gynaecologists. Karachi doctors should be educated in the correct management and counselling of STIs to prevent further spread of STIs including AIDS.

(Sex Transm Inf 2000;76:383-385)

Keywords: sexually transmitted infections; general practitioners; attitudes and practices

\section{Introduction}

Pakistan is thought to have a low prevalence but a high risk of HIV. ${ }^{1}$ Sexually transmitted infections (STIs) increase the risk of HIV transmission twofold to sixfold. ${ }^{2}$ Thus, control and prevention of STIs is an important strategy for preventing HIV.

Physicians have a vital role in interrupting the spread of STIs through early diagnosis, appropriate treatment, and counselling regarding further prevention. This study was conducted to assess the knowledge, attitude and practices regarding STIs among general practitioners (GPs) and specialists in Karachi, Pakistan.

\section{Methods}

STUDY POPULATION AND SAMPLING METHODS We selected hospitals in Karachi that had more than 100 inpatient beds and a separate outpatient department. We approached all the gynaecologists, urologists, and dermatologists practising at these facilities. A pharmaceutical company, which marketed products used for STI treatment, provided us with a list of the gynaecologists, urologists, dermatologists, and general practitioners (GPs) whose work addresses were within $10 \mathrm{~km}$ of the Aga Khan University. We approached each of these practitioners.
We classified doctors as specialists if they had received postgraduate training in their respective specialties and GPs were licensed doctors who had not received any formal postgraduate training.

\section{MATERIALS}

Interviewers administered a 39 item structured questionnaire.

STATISTICAL METHODS AND ANALYSIS

We classified doctors as seeing and treating STI patients if he/she saw at least one STI patient per month.

Treatment responses (drug choice and dose/ duration of administration) were considered correct if they coincided with the current WHO or US Centers for Disease Control and Prevention (CDC) criteria for the treatment of STIs. ${ }^{34}$

We compared the difference between groups using relative risks and evaluated the potential of chance using $\chi^{2}$. We considered a $p$ value of $<0.05$ as statistically significant. All analysis was carried out using EPI-INFO. ${ }^{5}$

\section{Results}

We approached 120 doctors; 100 (83\%) completed the questionnaire including 46 GPs, 
Table 1 Comparison of the knowledge of Karachi general practitioners and specialists in the treatment of different STIs, 1998

\begin{tabular}{|c|c|c|c|c|c|}
\hline & $\begin{array}{l}\text { No (\%) of specialists } \\
\text { who answered correctly * }\end{array}$ & $\begin{array}{l}\text { No (\%) of general } \\
\text { practitioners who } \\
\text { answered correctly }\end{array}$ & Relative risk & $\begin{array}{l}95 \% \text { Confidence } \\
\text { interval }\end{array}$ & $p$ Value \\
\hline Appropriate antimicrobials for gonorrhoea & $28(52)$ & $22(48)$ & 1.1 & $0.7,1.6$ & 0.688 \\
\hline Appropriate dose and duration of antimicrobials for gonorrhoea & $15(54)$ & $8(36)$ & 1.5 & $0.8,2.8$ & 0.226 \\
\hline $\begin{array}{l}\text { Treatment of both gonorrhoea and chlamydia for mucopus in } \\
\text { cervix/urethra }\end{array}$ & $28(54)$ & $14(33)$ & 1.7 & $1.0,2.7$ & 0.038 \\
\hline Appropriate antimicrobials for chlamydia & $39(72)$ & $19(41)$ & 1.8 & $1.2,2.6$ & 0.002 \\
\hline Appropriate dose and duration of antimicrobials for chlamydia & $22(56)$ & $6(32)$ & 1.8 & $0.9,3.7$ & 0.076 \\
\hline Appropriate antimicrobials for syphilis & $43(80)$ & $22(49)$ & 1.6 & $1.2,2.3$ & 0.001 \\
\hline Appropriate dose and duration of antimicrobials for syphilis & $22(51)$ & $2(9)$ & 5.6 & $1.5,21.8$ & 0.001 \\
\hline Diagnosis of painful genital vesicular lesions as herpes genitalis & $38(72)$ & $13(28)$ & 2.5 & $1.6,4.1$ & 0.000 \\
\hline Appropriate antimicrobials for herpes genitalis & $35(90)$ & $8(62)$ & 1.5 & $1.0,2.3$ & 0.009 \\
\hline Appropriate dose and duration of antimicrobials for herpes genitalis & $15(43)$ & $5(63)$ & 0.7 & $0.4,1.3$ & 0.315 \\
\hline
\end{tabular}

^In accordance with international guidelines. Percentages are out of the total responses received.

23 dermatologists, 21 gynaecologists, and 10 urologists.

Of the 100 interviewed doctors, 80 had seen at least one new STI patient in the preceding month. The mean number of STI patients seen per month by specialty were dermatologists 15 , gynaecologists 13, urologists six, and GPs four patients.

When asked, using an open ended question, what the most common STI they saw in their practice $53 \%$ of gynaecologists responded vaginal candidiasis, a syndrome that is not even an STI. The other physicians reported gonorrhoea as most common.

KNOWLEDGE

We asked all the doctors questions regarding knowledge of STIs. Given a patient presenting with mucopus in the cervix/urethra, 42 (44\%) said that they would treat for both gonorrhoea and chlamydia, $38(40 \%)$ would treat for gonorrhoea only, $10(10 \%)$ would treat for chlamydia only, while five (5\%) would treat for syphilis. Specialists were consistently more likely to answer questions in accordance with international guidelines for diagnosis and treatment of STIs than were general practitioners. However, even among specialists, less than half were able to correctly state the dose and duration of antimicrobial treatment for the most common STIs (table 1). Among specialists, compared with gynaecologists, urologists and dermatologists were 2.5 times more likely to report the appropriate dose and duration of penicillin for syphilis ( $61 \%$ versus $25 \%$, relative risk $2.5, \mathrm{p}=0.03$ ), and 1.9 times more likely to report the appropriate antimicrobials for treatment of gonorrhoea ( $64 \%$ versus $33 \%$, relative risk $1.9, \mathrm{p}=0.03$ ).

The doctors listed their major sources of knowledge regarding STIs as medical training $(52 \%)$, medical journals $(50 \%)$, and continuous medical education (30\%)

PRACTICES

We asked only those physicians who saw STI patients (80), questions regarding STI treatment practices. Twenty five doctors (31\%) reported that they diagnosed STIs using only history and physical examination, while 54 doctors $(68 \%)$ based their diagnosis on clinical evaluation and microscopy and/or culture.

Thirty one doctors (39\%) never screened STI patients for HIV and $17(21 \%)$ sometimes did. The most common reasons for not screen- ing were because the patients could not afford the test $(n=16,42 \%)$, the history was not suggestive $(n=13,34 \%)$, and HIV was not prevalent in Pakistan $(n=13,34 \%)$.

Sixty two doctors $(78 \%)$ asked their patients about their number of sexual partners; 54 $(68 \%)$ asked about the use of barrier methods; $68(85 \%)$ advised their patient to use condoms. Thirty three doctors $(41 \%)$ encouraged STD patients to inform their partners about risk to them and $28(35 \%)$ asked the patient to bring in their partners.

\section{ATTITUDES}

Eighty five doctors (85\%) thought that Pakistan was at risk of having an AIDS outbreak. Eighty seven doctors $(87 \%)$ believed that control of STIs would decrease the risk of HIV transmission.

Thirty six per cent of the doctors believed that STI patients had loose morals; $21 \%$ believed that STI patients could not give up their careless sexual behaviour; while $43 \%$ believed that STI patients had other bad habits like drug addictions.

When asked what the single most important factor in the control of AIDS in Pakistan was, $81 \%$ answered mass media (radio, television, and newspapers) while $13 \%$ thought that education by doctors was the most effective way.

Ninety two per cent of the doctors were interested in attending continuing medical education seminars on STI control, and 95\% reported being willing to follow a standardised protocol for the management and counselling of STI patients.

\section{Discussion}

Karachi physicians, both GPs and specialists, lacked sufficient knowledge of STI symptomatology and therapy to diagnose and treat STI patients appropriately. When presented with a hypothetical cases of common STIs-syphilis, chlamydia, and gonorrhoea-the majority of cases were incorrectly diagnosed, and the drug dose and duration for the STIs questioned was, more than half the time, inappropriate.

Compared to the GPs, specialists were more likely to diagnose STDs correctly, and to prescribe the appropriate drugs at appropriate dosages. This is probably because the GPs in Karachi do not receive any formal postgraduate training in the management of STD patients, and very little emphasis is placed on 
STDs during undergraduate training, since Karachi is believed to be an area of low prevalence.

Urologists and dermatologists were more likely to correctly diagnose and treat most STIs than the gynaecologists. Since most female STI patients in Karachi present to gynaecologists, these data suggest a specific need to improve STI treatment and care among women.

An important limitation of this study is that the clinic based doctors were selected from a list provided by a pharmaceutical company from a single section of the city, and therefore may not be representative of all Karachi physicians. However, the physicians listed by the pharmaceutical companies included those who saw the largest number of patients and so represented major providers of STD care available in Karachi.

With a relatively low HIV prevalence in Pakistan, it is a particularly appropriate time to invest in prevention. Postgraduate training of physicians in STI control improves STI management. ${ }^{6}$ The data from this study demonstrate both the need for such education, and the physicians' willingness to participate. It should be a high priority for HIV prevention in Pakistan.

We are grateful to Naeem Rahim, Amana Nasir, Sumbal Khalid, Khalid Nuri, Aida Khanum, and Shakeel Thakurdas for their contribution in data collection.

The project was funded by the Department of Community Health Sciences, the Aga Khan University, Karachi.

Contributors: HEK was the primary author of the study protocol, assisted in development of the questionnaires, and drafted the manuscript; SL reviewed each draft of the protocol, questionnaire and manuscript, framed the analysis and outlined the manuscript; SR assisted in the development of the protocol manuscript.

1 Laqa M. Epidemiology and control of STDs in developing countries. Sex Transm Dis 1994;21(Suppl).

2 Laga M, Diallo MO, Bune A. Inter-relationship of STD and HIV. AIDS 1994, a year in review.

3 Centers for Disease Control and Prevention. Sexually transmitted disease guide 1993. Atlanta: CDC.

4 Drugs used in STDs and HIV infection. WHO model prescribing information. Geneva: WHO, 1995.

5 Dean AG, Dean JA, Coulombier D, et al. Epi Info, Version 6.04. Atlanta, GA: Centers for Disease Control and Prevention, USA, 1995.

6 Gully PR, Fisher DC, Pless R, et al. How well do family physicians manage sexually transmitted diseases? Can Fam Physician 1995;41:1890-6. 